\title{
Note on the asymptotic structure of Kerr-Schild form
}

\author{
Pujian Mao and Weicheng Zhao \\ Center for Joint Quantum Studies and Department of Physics, School of Science, \\ Tianjin University, 135 Yaguan Road, Tianjin 300350, China \\ E-mail: pjmao@tju.edu.cn, zhaoweichengok@tju.edu.cn
}

ABSTRACT: The Kerr-Schild form provides a natural way of realizing the classical double copy that relates exact solutions in general relativity to exact solutions in gauge theory. In this paper, we examine the asymptotic structure of Kerr-Schild form. In Newman-Unti gauge, we find a generic solution space satisfying the Kerr-Schild form in series expansion around null infinity. The news function in the solution space is chiral and can not lead to a mass loss formula. A class of asymptotically flat complex pp-wave solutions in closed form is obtained from the solution space.

Keywords: Classical Theories of Gravity, Scattering Amplitudes

ArXiv EPrint: 2109.09676 


\section{Contents}

1 Introduction 1

2 The Newman-Unti gauge 3

3 The Kerr-Schild form $\quad 4$

4 The solution space $\quad 5$

4.1 Hypersurface equations 5

$\begin{array}{lll}4.2 & \text { Standard equations } & 6\end{array}$

$\begin{array}{lll}4.3 & \text { Solution space in series expansion } & 6\end{array}$

4.4 Supplementary equations 6

4.5 The Kerr-Shcild constraint $\quad 7$

4.6 Exact solution 8

$\begin{array}{llr}5 & \text { Discussion } & 9\end{array}$

\section{Introduction}

The double copy structure $[1,2]$ reveals a remarkable relation between gravity and gauge theory in the context of scattering amplitudes. Apart from the fruitful results and applications (see, e.g., [3] for a review and references therein), it is still somewhat mysterious that what is the interpretation of double copy from the field theory side, in particular at the classical level. In a pioneering paper [4], an exact classical double copy was demonstrated for a particular class of solutions in gravity that admits a Kerr-Schild form. Shortly, this type of classical double copy has been extensively investigated and developed [5-43].

The success of Kerr-Schild double copy is temporarily subject to algebraically special solutions. One of the remarkable features of the Kerr-Schild metric (see, e.g., [44] for detailed introduction) is the fact that the null vector and the scalar field in the decomposition can be interpreted as perturbations on the flat background. Such a property is very crucial in connecting solutions of gravitational theory to gauge theory. Given the fact that the numbers of known exact solutions of gravity are indeed limited, in particular the ones admitting a Kerr-Schild form in four dimensional Einstein gravity, the connection between gravity and gauge theory in the Kerr-Schild double copy formalism is very restricted. However, if one considers solutions of Einstein equation in a perturbative way, namely, given in series expansion, there can be a solution space including infinite amount of solutions. In the seminal work by Bondi et. al. [45], it is shown that when expanding the metric fields in inverse powers of a radius coordinate in asymptotic region, the Einstein equation is formulated as a characteristic initial value problem. In this framework, the gravitational 
radiation is characterized by the news functions. Once reasonable news functions and initial data are given, a solution of Einstein equation is determined. Naturally one would wonder that if the asymptotic framework can allow us to extend the application of classical double copy.

Indeed, such idea has already been implemented in literature recently. In [42], the authors consider the rotating STU supergravity black holes [46], which are only algebraically special asymptotically. The classical double copy is confirmed at the leading order in the asymptotic expansion. In [43], a precise prescription is given to connect asymptomatically flat gauge and gravitational fields. In this paper, we start from an alternative point of view. We adopt the Kerr-Schild form in the asymptotic framework and study the solution space admitting Kerr-Schild form in asymptotic expansion around null infinity in four dimensional spacetime. This computation is also motivated by the triangle relation [47] where the scattering amplitudes in the low energy limit, i.e., soft theorems, are connected to asymptotic symmetries and memory effects. Clearly the soft theorems obey a double copy structure that the soft graviton theorem is double the soft photon one. While the study of asymptotic symmetry and memories are mostly done in the region around null infinity. The Newman-Unti (NU) gauge [48] is adopted to derive the solution space.

Because of the constraint from the Kerr-Schild form, the solutions in NU gauge satisfying the real condition must be spherically symmetric. Then Birkhoff's theorem implies that the only vacuum solution is Schwarzschild. Nevertheless, we continue to derive a solution space by removing the real condition. There are many reasons to explore complex solutions of Einstein equation, in particular in the context of classical double copy. The complex solutions in the present work are real solutions in the split metric signature $(+,+,-,-)$. The self-dual sector in light-cone coordinates with split signature has very important applications in classical double copy $[4,13,31,33]$. Other applications of complex solutions can be found, e.g., in [49-51]. In the stereographic coordinates, we find a self-consistent solution space. The news function and the initial data are constrained from the Kerr-Schild form. It is difficult to give a generic solution to the Kerr-Schild constraint. Alternatively, we provide two simple solutions for the news function and leading initial data. Those two cases correspond to two classes of solutions. It is worthwhile to emphasize that any solution of the news function and the initial data to the Kerr-Schild constraint will give a solution of vacuum Einstein equation. We also find an interesting solution in closed form. It is pp-wave solution and is a linear superposition of self-dual and anti-self-dual solutions. In the complex case, the news function is chiral. Consequently, it can not lead to a mass loss formula which is not surprising in the sense that the radiation taking away the energy from the system should satisfy real condition.

The organization of this paper is as follows: In the next section, we give a brief review of the NU gauge. In section 3, we combine the NU gauge with the Kerr-Schild form to fix the gauge conditions for the present work. Section 4 is devoted to the deviation of the solution space satisfying the gauge conditions. Exact solutions in the anti-self-dual or the self-dual sectors are also provided in this section. We close in the last section with some remarks.

Note added: when writing up this paper, we became aware of $[42,43]$ with overlapping motivations with ours. 


\section{The Newman-Unti gauge}

The retarded coordinates $(u, r, z, \bar{z})$ is the most convenient choice of the coordinates system to work with physical fields at future null infinity where $(z, \bar{z})$ is the complex stereographic coordinates that is related to the usual angular variables $(\theta, \phi)$ by $z=\cot \frac{\theta}{2} e^{i \phi}$. The NU gauge was originally introduced in the Newman-Penrose formalism [52] to study the asymptotic behavior of Weyl tensor and metric tensor. Adapted to metric formalism, the metric and inverse metric have the form [48]

$$
g_{\mu \nu}=\left(\begin{array}{cccc}
g_{u u} & -1 & g_{u z} & g_{u \bar{z}} \\
-1 & 0 & 0 & 0 \\
g_{u z} & 0 & g_{z z} & g_{z \bar{z}} \\
g_{u \bar{z}} & 0 & g_{z \bar{z}} & g_{\bar{z} \bar{z}}
\end{array}\right), \quad g^{\mu \nu}=\left(\begin{array}{cccc}
0 & -1 & 0 & 0 \\
-1 & g^{r r} & g^{r z} & g^{r \bar{z}} \\
0 & g^{r z} & g^{z z} & g^{z \bar{z}} \\
0 & g^{r \bar{z}} & g^{z \bar{z}} & g^{\bar{z} \bar{z}}
\end{array}\right)
$$

For computational simplicity, we map the celestial sphere at null infinity to a $2 \mathrm{~d}$ plane. The connection between those two choices can be found, for instance, in [53-56]. The line element of the Minkowski spacetime now is

$$
\mathrm{d} s^{2}=-2 \mathrm{~d} u \mathrm{~d} r+2 r^{2} \mathrm{~d} z \mathrm{~d} \bar{z} .
$$

The asymptotic behaviors of the non-vanishing metric components preserving asymptotic flatness are required as

$$
\begin{aligned}
& g_{u u}=\mathcal{O}\left(r^{-1}\right), \quad g_{u z}=\mathcal{O}(1), \quad g_{u \bar{z}}=\mathcal{O}(1), \\
& g_{z z}=\mathcal{O}(r), \quad g_{\bar{z} \bar{z}}=\mathcal{O}(r), \quad g_{z \bar{z}}=r^{2}+\mathcal{O}(1) \text {. }
\end{aligned}
$$

To solve the Einstein equation, it is very useful to arrange all components as follows:

- Four hypersurface equations:

$$
G_{r \mu}=0
$$

- Two standard equations:

$$
G_{z z}=G_{\bar{z} \bar{z}}=0
$$

- One trivial equation:

$$
G_{z \bar{z}}=0
$$

- Three supplementary equations:

$$
G_{u z}=G_{u \bar{z}}=G_{u u}=0 .
$$

The Bianchi identities can be written as

$$
\nabla_{\mu} G^{\mu}{ }_{\nu}=\frac{1}{\sqrt{-g}} \partial_{\mu}\left(\sqrt{-g} G^{\mu}{ }_{\nu}\right)+\frac{1}{2} G_{\mu \rho} \partial_{\nu} g^{\mu \rho}=0 .
$$

When the hypersurface equations and standard equations are satisfied, the Bianchi identity $\nabla_{\mu} G^{\mu}{ }_{r}=0$ yields

$$
G_{z \bar{z}} \partial_{r} g^{z \bar{z}}=0
$$


Hence $G_{z \bar{z}}=0$ because of the boundary condition for $g_{z \bar{z}}$, which means that the trivial equation is satisfied automatically. Then one obtains that $R=0$. The Bianchi identity $\nabla_{\mu} G^{\mu}{ }_{z}=0$ reduces to $\partial_{r}\left(\sqrt{-g} G_{u z}\right)=0$, noticing that the hypersurface equations and standard equations are satisfied. Similarly, the Bianchi identity $\nabla_{\mu} G^{\mu} \bar{z}=0$ yields $\partial_{r}\left(\sqrt{-g} G_{u \bar{z}}\right)=0$. The last Bianchi identity $\nabla_{\mu} G^{\mu}{ }_{u}=0$ leads to $\partial_{r}\left(\sqrt{-g} G_{u u}\right)=0$ when the rest equations are satisfied. Hence three supplementary equations are only left with only one order to solve in the asymptotic expansion.

\section{The Kerr-Schild form}

A metric of Kerr-Schild form is obtained from a deformation of a fixed background metric $\bar{g}_{\mu \nu}$. In the retarded coordinates $(u, r, z, \bar{z})$, we choose the background metric as the Minkowski spacetime (2.2). The deformation is made by a null vector field $k_{\mu}$ and a scalar function $\phi$ in the form

$$
g_{\mu \nu}=\eta_{\mu \nu}+\phi k_{\mu} k_{\nu}
$$

One can show that $k^{\mu}$ is null when contracted with both the full metric $g_{\mu \nu}$ and the Minkowski one $\eta_{\mu \nu}$. Therefore, the inverse metric is just

$$
g^{\mu \nu}=\eta^{\mu \nu}-\phi k^{\mu} k^{\nu}
$$

Hence we can raise the index of $k$ using both $g^{\mu \nu}$ and $\eta^{\mu \nu}$. In the NU gauge, it is required that

$$
g_{r r}=g_{r z}=g_{r \bar{z}}=0, \quad g_{u r}=-1 .
$$

Combining these conditions with the Kerr-Schild ansatz (3.1), one should require that $k_{r}=0$. Because $k_{\mu}$ is a null vector respect to $\eta^{\mu \nu}$, the solution of the remaining components is either $k_{z}=0$ or $k_{\bar{z}}=0$. It is important to point out that such solution will not lead to a real metric. In other works, the only solution in the usual real angular variables $(\theta, \phi)$ is the trivial one $k_{\theta}=k_{\phi}=0$. However, it is still meaningful to continue the computation in the complex coordinates with the chiral solution. On the one hand, the self-dual metric fields in the retarded coordinates $(u, r, z, \bar{z})$ are exactly in this form. The self-dual gravity is a very important realization of the classical double copy $[4,13,31,33]$. Other important applications of self-dual gravity can be found, e.g., in [57-59] and references therein. On the other hand, this situation is somehow similar to the gluon three point amplitude at tree level. As the smallest or starting point amplitude in the BCFW recursion relation [60], it is vanishing for real null momenta. Alternatively, one works with complex momenta where the helicity spinor $\lambda_{i}$ and $\tilde{\lambda}_{i}$ are independent and the three-gluon amplitudes are derived from either left-handed spinors or right-handed spionrs, see, e.g., [61, 62] for a textbook treatment. We choose $k_{\bar{z}}=0$ in the present work. Substituting this solution back to the metric, one obtains

$$
g_{u \bar{z}}=0=g_{\bar{z} \bar{z}} .
$$

The remaining components of the metric have the form

$$
g_{u u}=\phi k_{u} k_{u}, \quad g_{z z}=\phi k_{z} k_{z}, \quad g_{u z}=\phi k_{u} k_{z} .
$$


Thus they should satisfy the relation as follows

$$
g_{u u} g_{z z}=g_{u z}^{2} .
$$

The aim of the present work is to check the constraints from the Kerr-Schild form (3.4) and (3.6) on the solution space in NU gauge. Before doing so, we would like to point out that a double Kerr-Schild form

$$
g_{\mu \nu}=\eta_{\mu \nu}+\phi k_{\mu} k_{\nu}+\Psi l_{\mu} l_{\nu}
$$

where $k^{2}=l^{2}=k \cdot l=0$, can not bring the other half copy of the metric. The NU gauge condition will fix both $k_{r}=0$ and $l_{r}=0$. The null conditions of $k_{\mu}$ and $l_{\mu}$ bring the same constraint as single Kerr-Schild form. Let us choose $k_{\bar{z}}=0$ and $l_{z}=0$ to keep both components $g_{z z}$ and $g_{\bar{z} \bar{z}}$ in the metric. But the last condition that $k \cdot l=0$ eventually leads to $k_{z} l_{\bar{z}}=0$. Hence we are back to the single Kerr-Schild form.

\section{The solution space}

Once the gauge condition from the Kerr-Schild is clarified, the solution of Einstein equation in the retarded coordinates can be derived in a straightforward way following the computation in $[45,63-66]$. We start with the hypersurface equations which determine the radial dependence of the metric from the initial data. The standard equations control the time evolutions of the initial data. Then the time evolution of the integration constants from the hypersurface equations are fixed by the supplementary equations. In this work, there is one more constraint from the Kerr-Schild form.

\subsection{Hypersurface equations}

The hypersurface equations $G_{r r}=0$ and $G_{r \bar{z}}=0$ are satisfied once the Kerr-Schild form is applied by imposing (3.4). The next one $G_{r z}=0$ yields

$$
\partial_{r}\left(r^{4} \partial_{r}\left(\frac{g_{u z}}{r^{2}}\right)\right)=r^{2} \partial_{r}\left(\frac{\partial_{\bar{z}} g_{z z}}{r^{2}}\right) .
$$

Hence $g_{u z}$ can be solved out from $g_{z z}$ as

$$
g_{u z}=\frac{N(u, z, \bar{z})}{r}+r^{2} \int_{r}^{\infty} \mathrm{d} r^{\prime} \frac{1}{r^{\prime}} \int_{r^{\prime}}^{\infty} \mathrm{d} r^{\prime \prime} r^{\prime \prime 2} \partial_{r^{\prime \prime}}\left(\frac{\partial_{\bar{z}} g_{z z}}{r^{\prime \prime 2}}\right)
$$

where function $N(u, z, \bar{z})$ is an integration constant in $r$. We continue with $G_{u r}=0$, from which one obtains

$$
\partial_{r}\left(r g_{u u}\right)=\frac{1}{2 r^{2}}\left[\partial_{r}\left(r^{2} \partial_{\bar{z}} g_{u z}\right)-\partial_{\bar{z}}^{2} g_{z z}\right] .
$$

Thus $g_{u u}$ is fixed as

$$
g_{u u}=\frac{M(u, z, \bar{z})}{r}-\frac{1}{r} \int_{r}^{\infty} \mathrm{d} r^{\prime} \frac{1}{2 r^{\prime 2}}\left[\partial_{r^{\prime}}\left(r^{\prime 2} \partial_{\bar{z}} g_{u z}\right)-\partial_{\bar{z}}^{2} g_{z z}\right],
$$

where $M(u, z, \bar{z})$ is another integration constant. 


\subsection{Standard equations}

Equation $G_{\bar{z} \bar{z}}=0$ is satisfied automatically because of the Kerr-Schild form. The other standard equation leads to

$$
\begin{aligned}
\partial_{u} \partial_{r}\left(\frac{g_{z z}}{r}\right)= & \frac{1}{2 r^{4}}\left[4 r g_{u z}^{2}+2 g_{z z} \partial_{\bar{z}} g_{u z}+4 r^{2} g_{z z} \partial_{r} g_{u u}+r \partial_{\bar{z}} g_{z z} \partial_{r} g_{u z}\right. \\
& +r^{3}\left(\partial_{r} g_{u z}\right)^{2}-r \partial_{\bar{z}} g_{u z} \partial_{r} g_{z z}-r^{3} \partial_{r} g_{u u} \partial_{r} g_{z z} \\
& -2 r g_{z z} \partial_{r} \partial_{\bar{z}} g_{u z}-g_{u z}\left(4 \partial_{\bar{z}} g_{z z}+4 r^{2} \partial_{r} g_{u z}-2 r \partial_{r} \partial_{\bar{z}} g_{z z}\right) \\
& \left.+2 r^{3} \partial_{r} \partial_{z} g_{u z}+r^{3} g_{z z} \partial_{r}^{2} g_{u u}-r g_{u u}\left(2 g_{z z}-2 r \partial_{r} g_{z z}+r^{2} \partial_{r}^{2} g_{z z}\right)\right] .
\end{aligned}
$$

Clearly, there is no constraint at the order $\mathcal{O}(r)$ of $g_{z z}$ from the standard equation. The field at this order is related to the news function in the system which indicates propagating degree of freedom.

\subsection{Solution space in series expansion}

Suppose that $g_{z z}$ is given as initial data as ${ }^{1}$

$$
g_{z z}=c(u, z, \bar{z}) r+f_{0}(u, z)+\sum_{i=1}^{\infty} \frac{f_{i}(u, z, \bar{z})}{r^{i}} .
$$

Inserting the initial data into (4.2) and (4.4), one obtains

$$
g_{u z}=\frac{1}{2} \partial_{z} c+\frac{N}{r}-\sum_{i=1}^{\infty} \frac{i+2}{i(i+3)} \frac{\partial_{\bar{z}} f_{i}}{r^{i+1}},
$$

and

$$
g_{u u}=\frac{M}{r}-\frac{\partial_{\bar{z}} N}{2 r^{2}}+\sum_{i=1}^{\infty} \frac{1}{i(i+3)} \frac{\partial_{\bar{z}}^{2} f_{i}}{r^{i+2}} .
$$

\subsection{Supplementary equations}

With the series expansion, one can continue to solve the supplementary equations. From $G_{u z}=0$, we obtain

$$
\partial_{u} N=\frac{1}{3} \partial_{z} M-\frac{1}{6} \partial_{z} \partial_{\bar{z}}^{2} c .
$$

Next, $G_{u \bar{z}}=0$ yields

$$
2 \partial_{\bar{z}} M+\partial_{\bar{z}}^{3} c=0 .
$$

The last supplementary equation $G_{u u}=0$ leads to

$$
\partial_{u} M=\frac{1}{2} \partial_{u} \partial_{\bar{z}}^{2} c
$$

It is of convenience to define

$$
M_{0}=M-\frac{1}{2} \partial_{\bar{z}}^{2} c .
$$

\footnotetext{
${ }^{1}$ The absent of $\bar{z}$-dependence in $f_{0}(u, z)$ is to avoid logarithmic terms which will involve a polyhomogeneous solution space.
} 
Then (4.11) and (4.9) reduce to

$$
\partial_{u} M_{0}=0, \quad \partial_{u} N=\frac{1}{3} \partial_{z} M_{0} .
$$

Hence $M_{0}$ is independence of $u$ and $N=N_{0}(z, \bar{z})+\frac{1}{3} u \partial_{z} M_{0}$. So we do not have a mass loss formula in this system as $M_{0}$ is not changing. Moreover, (4.10) becomes

$$
\partial_{\bar{z}} M_{0}+\partial_{\bar{z}}^{3} c=0 .
$$

Since $M_{0}$ is independence of $u$, one can have that $\partial_{u} \partial_{\bar{z}}^{3} c=0$. Therefore, a generic solution to $c$ should have the form

$$
c=\tilde{c}(z, \bar{z})+c_{0}(u, z)+c_{1}(u, z) \bar{z}+c_{2}(u, z) \bar{z}^{2} .
$$

Hence

$$
M_{0}=\bar{M}(z)-\partial_{\bar{z}}^{2} \tilde{c}, \quad M=\bar{M}-\frac{1}{2} \partial_{\bar{z}}^{2} \tilde{c}+c_{2}, \quad N=N_{0}+\frac{1}{3} u\left(\partial_{z} \bar{M}-\partial_{z} \partial_{\bar{z}}^{2} \tilde{c}\right) .
$$

\subsection{The Kerr-Shcild constraint}

The first two constraints in (3.4) from the Kerr-Schild form have been implemented when solving the Einstein equation. The last one in (3.6) can be considered as a constraint on the initial data. In the series expansion (4.6), the fields from each order are constrained from both the standard equation (4.5) and the Kerr-Schild form (3.6). For the first order, the news aspect $c$ is only constrained from the Kerr-Schild form as

$$
c M=\frac{1}{4}\left(\partial_{\bar{z}} c\right)^{2},
$$

For the second order, one obtains

$$
\begin{aligned}
& \partial_{u} f_{0}=0, \\
& f_{0} M=N \partial_{\bar{z}} c+\frac{1}{2} c \partial_{\bar{z}} N .
\end{aligned}
$$

Clearly, the first equation shows that $f_{0}$ has only $z$ dependence. Hence the second equation will lead to another constraint on $c$. Though it is not easy to give a generic solution for $c$, one can easily find some non trivial solutions, e.g.,

$$
f_{0}=M_{0}=N_{0}=\tilde{c}=c_{0}=c_{1}=0, \quad M=c_{2}(u, z), \quad c=c_{2}(u, z) \bar{z}^{2} .
$$

and

$$
M=\tilde{c}=c_{1}=c_{2}=0, \quad N=N_{0}(z), \quad c=c_{0}(u, z) .
$$

We can continue to trace higher orders. For every $f_{i}(i \geq 1)$, there are two differential equations. Considering $f_{1}$ as example, one has

$$
\begin{gathered}
2 f_{0} \partial_{\bar{z}} N-3 \partial_{\bar{z}} f_{1} \partial_{\bar{z}} c-c \partial_{\bar{z}}^{2} f_{1}-4 f_{1} M+4 N^{2}=0 \\
\partial_{u} f_{1}=\frac{1}{8} c\left(2 M-\partial_{\bar{z}}^{2} c\right)+\frac{1}{2} \partial_{z} N
\end{gathered}
$$


Again, a generic solution for $f_{1}$ is very to find once the other fields in the equations are known. One can show that there is non trivial solution when the other fields are given as (4.20). A simple one is $f_{1}=\frac{\tilde{f}_{1}(z)}{\bar{z}}$ where $\tilde{f}_{1}(z)$ is an arbitrary function of $z$. Hence, one can expect that there should not be more constraint on the news aspect $c$ from the equations at higher orders. Since the dynamics is only encoded in the news aspect $c$, the equations from higher orders will not bring any new information.

\subsection{Exact solution}

There is a simple but very interesting solution in closed form in the solution space. The metric is given by

$$
\mathrm{d} s^{2}=-2 \mathrm{~d} u \mathrm{~d} r+2 r^{2} \mathrm{~d} z \mathrm{~d} \bar{z}+r[c(u, z)+f(r, z)] \mathrm{d} z^{2} .
$$

This solution is non-trivial in the sense that it has non vanishing components of Riemann tensor which are

$$
R_{u z u z}=-\frac{1}{2} r \partial_{u}^{2} c, \quad R_{r z r z}=-\frac{1}{2} r \partial_{r}^{2} f .
$$

The square of the Riemann tensor $R_{\mu \nu \alpha \beta} R^{\mu \nu \alpha \beta}$ is zero. The vector $k=\frac{\partial}{\partial \bar{z}}$ is null and tangent to an expansion-free, shear-free and twist-free null geodesic congruence, i.e., satisfying $\nabla_{\mu} k_{\nu}=0$. So this solution belongs to the class of pp-wave solutions following the definition in $[67,68]$. Interestingly, this pp-wave solution is asymptotically flat when the asymptotic behavior of $f(r, z)$ is $\mathcal{O}\left(\frac{1}{r}\right)$ which is a direct consequence of the NU setup in section 2 with a special boundary choice (2.2), i.e., a 2 d plane. Coordinate $z$ labels the null wave surfaces and coordinates $u$ and $r$ span the wave surfaces. There is another null direction $l=\frac{\partial}{\partial r}$ that has expansion and shear. With the following change of coordinates

$$
\bar{z}=\rho, \quad z=-v, \quad r=\frac{1}{\zeta}, \quad u=\bar{\zeta},
$$

the line element (4.24) becomes

$$
\mathrm{d} s^{2}=\frac{1}{\zeta^{2}}\left[-2 \mathrm{~d} v \mathrm{~d} \rho-2 H(v, \zeta, \bar{\zeta}) \mathrm{d} v^{2}+2 \mathrm{~d} \zeta \mathrm{d} \bar{\zeta}\right],
$$

where $H(v, \zeta, \bar{\zeta})=-\frac{1}{2} \zeta[c(v, \bar{\zeta})+f(v, \zeta)]$. This metric is conformal to the Brinkmann form [68] with a complex conformal factor. This solution might be considered as a generalization of the Brinkmann form to the complex case. Writing the line element (4.24) in a more generic form as

$$
\mathrm{d} s^{2}=-2 \mathrm{~d} u \mathrm{~d} r+2 r^{2} \mathrm{~d} z \mathrm{~d} \bar{z}+r H(u, r, z) \mathrm{d} z^{2},
$$

the vacuum Einstein yields

$$
\partial_{u} \partial_{r} H(u, r, z)=0 \text {. }
$$

The precise choice in (4.24) is just the general solution to this equation.

By choosing four null basis as

$$
e_{\mu}^{1}=(0,1,0,0), \quad e_{\mu}^{2}=(1,0,0,0), \quad e_{\mu}^{3}=(0,0, r, 0), \quad e_{\mu}^{4}=\left(0,0, \frac{H}{2}, r\right),
$$


the solution can be described in a Newman-Penrose formalism. The non-vanishing Newman-Penrose variables are

$$
\begin{aligned}
\Psi_{0} & =\frac{\partial_{r}^{2} H}{2 r}, & \bar{\Psi}_{4} & =\frac{\partial_{u}^{2} H}{2 r}, \\
\rho & =\bar{\rho}=\frac{1}{r}, & \sigma & =-\frac{H-r \partial_{r} H}{2 r^{2}}, \quad \bar{\lambda}=-\frac{\partial_{u} H}{2 r} .
\end{aligned}
$$

Since (4.29) is a linear equation, a new solution can be obtained simply by superposing distinct solutions with different expressions for $H$ just like the case of pp-wave in Brinkmann form. An interesting feature of the solution (4.24) is the fact that it can be considered as a linear superposition of self-dual and anti-self-dual solutions. The case $c=0$ and $f \neq 0$ represents a self-dual solution while the other case $c \neq 0$ and $f=0$ represents an anti-selfdual solution. To see that, we define the spinorial tetrad as

$$
\sigma_{\mu_{A \dot{A}}}=\sigma_{A \dot{A}}^{a} e_{\mu}^{b} \eta_{a b},
$$

where

$$
\eta_{a b}=\eta^{a b}=\left(\begin{array}{cccc}
0 & -1 & 0 & 0 \\
-1 & 0 & 0 & 0 \\
0 & 0 & 0 & 1 \\
0 & 0 & 1 & 0
\end{array}\right), \quad \sigma^{a}=\frac{1}{2}\left(\sigma^{2}+i \sigma^{3}, \sigma^{2}-i \sigma^{3}, \mathbb{1}-\sigma^{1}, \mathbb{1}+\sigma^{1}\right)
$$

$\sigma^{k}(k=1,2,3)$ are the Pauli matrices and the tetrad is chosen as (4.30). In our convention, $\varepsilon^{12}=1$. One can easily verify that

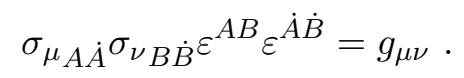

The spinoral form of the Weyl tensor is

$$
W_{A \dot{A} B \dot{B} C \dot{C} D \dot{D}}=C_{A B C D} \varepsilon_{\dot{A} \dot{B}} \dot{C}_{\dot{C} \dot{D}}+\bar{C}_{\dot{A} \dot{B} \dot{C} \dot{D}} \varepsilon_{A B} \varepsilon_{C D}
$$

$C_{A B C D}$ and $\bar{C}_{\dot{A} \dot{B} \dot{C} \dot{D}}$ are totally symmetric and they represent the anti-self-dual and selfdual parts of the curvature respectively. For a real metric, they are complex conjugation of each other. Direct computation regarding to the solution (4.24) gives

$$
C_{1111}=C_{1122}=C_{2222}=\frac{1}{8 r} \partial_{u}^{2} c, \quad C_{1112}=C_{1222}=-\frac{1}{8 r} \partial_{u}^{2} c
$$

and

$$
\bar{C}_{1111}=\bar{C}_{1122}=\bar{C}_{2222}=\frac{1}{8 r} \partial_{r}^{2} f, \quad \bar{C}_{1112}=\bar{C}_{1222}=-\frac{1}{8 r} \partial_{r}^{2} f .
$$

\section{Discussion}

We obtain a generic solution space in series expansion near null infinity in the NU gauge satisfying the Kerr-Schild form. The solutions only exist in complex coordinates which should correspond to a complex classical double copy. Interesting exact solutions in the 
anti-self-dual and the self-dual sectors can be read out from the solution space. As the single copy or zeroth copy version of the solution space, the null vector $k_{\mu}$ and the scalar field $\phi$ in the Kerr-Schild form can be easily derived from (3.5).

The solution space is self-contained. But the result itself somehow indicates the incompatibility between the NU gauge and Kerr-Schild form. The only non trivial real solution in NU gauge satisfying the Kerr-Schild form is the spherically symmetric one. With such shortcoming, applications of the present result in the triangle relation are now less clear. For instance, what is a memory effect in the Kerr-Schild form and what is the interpretation of the memory effect from the point of view of classical double copy structure. We do not yet have a clear answer to those questions and leave them for future investigation.

\section{Acknowledgments}

The authors thank Zhengwen Liu, Hong Lü, Jun-Bao Wu, and Xiaoning Wu for useful discussions. This work is supported in part by the National Natural Science Foundation of China under Grant No. 11905156 and No. 11935009.

Open Access. This article is distributed under the terms of the Creative Commons Attribution License (CC-BY 4.0), which permits any use, distribution and reproduction in any medium, provided the original author(s) and source are credited.

\section{References}

[1] Z. Bern, J.J.M. Carrasco and H. Johansson, New relations for gauge-theory amplitudes, Phys. Rev. D 78 (2008) 085011 [arXiv: 0805.3993] [INSPIRE].

[2] Z. Bern, J.J.M. Carrasco and H. Johansson, Perturbative quantum gravity as a double copy of gauge theory, Phys. Rev. Lett. 105 (2010) 061602 [arXiv:1004.0476] [INSPIRE].

[3] Z. Bern, J.J. Carrasco, M. Chiodaroli, H. Johansson and R. Roiban, The duality between color and kinematics and its applications, arXiv:1909.01358 [INSPIRE].

[4] R. Monteiro, D. O'Connell and C.D. White, Black holes and the double copy, JHEP 12 (2014) 056 [arXiv: 1410.0239] [INSPIRE].

[5] A. Luna, R. Monteiro, D. O'Connell and C.D. White, The classical double copy for Taub-NUT spacetime, Phys. Lett. B $\mathbf{7 5 0}$ (2015) 272 [arXiv:1507.01869] [INSPIRE].

[6] A. Luna, R. Monteiro, I. Nicholson, D. O'Connell and C.D. White, The double copy: Bremsstrahlung and accelerating black holes, JHEP 06 (2016) 023 [arXiv:1603.05737] [INSPIRE].

[7] C.D. White, Exact solutions for the biadjoint scalar field, Phys. Lett. B 763 (2016) 365 [arXiv: 1606.04724] [INSPIRE].

[8] N. Bahjat-Abbas, A. Luna and C.D. White, The Kerr-Schild double copy in curved spacetime, JHEP 12 (2017) 004 [arXiv:1710.01953] [INSPIRE].

[9] M. Carrillo-González, R. Penco and M. Trodden, The classical double copy in maximally symmetric spacetimes, JHEP 04 (2018) 028 [arXiv: 1711.01296] [INSPIRE]. 
[10] W.D. Goldberger, J. Li and S.G. Prabhu, Spinning particles, axion radiation, and the classical double copy, Phys. Rev. D 97 (2018) 105018 [arXiv:1712.09250] [INSPIRE].

[11] J. Li and S.G. Prabhu, Gravitational radiation from the classical spinning double copy, Phys. Rev. D 97 (2018) 105019 [arXiv: 1803.02405] [InSPIRE].

[12] K. Lee, Kerr-Schild double field theory and classical double copy, JHEP 10 (2018) 027 [arXiv: 1807.08443] [INSPIRE].

[13] D.S. Berman, E. Chacón, A. Luna and C.D. White, The self-dual classical double copy, and the Eguchi-Hanson instanton, JHEP 01 (2019) 107 [arXiv: 1809. 04063] [INSPIRE].

[14] M. Gurses and B. Tekin, Classical double copy: Kerr-Schild-Kundt metrics from Yang-Mills Theory, Phys. Rev. D 98 (2018) 126017 [arXiv:1810.03411] [InSPIRE].

[15] A. Luna, R. Monteiro, I. Nicholson and D. O'Connell, Type D spacetimes and the Weyl double copy, Class. Quant. Grav. 36 (2019) 065003 [arXiv:1810.08183] [INSPIRE].

[16] M. Carrillo González, B. Melcher, K. Ratliff, S. Watson and C.D. White, The classical double copy in three spacetime dimensions, JHEP 07 (2019) 167 [arXiv:1904.11001] [INSPIRE].

[17] W. Cho and K. Lee, Heterotic Kerr-Schild double field theory and classical double copy, JHEP 07 (2019) 030 [arXiv:1904.11650] [INSPIRE].

[18] A.P.V. and A. Manu, Classical double copy from color kinematics duality: a proof in the soft limit, Phys. Rev. D 101 (2020) 046014 [arXiv: 1907.10021] [INSPIRE].

[19] Y.F. Bautista and A. Guevara, On the double copy for spinning matter, JHEP 11 (2021) 184 [arXiv: 1908.11349] [INSPIRE].

[20] K. Kim, K. Lee, R. Monteiro, I. Nicholson and D. Peinador Veiga, The classical double copy of a point charge, JHEP 02 (2020) 046 [arXiv: 1912.02177] [INSPIRE].

[21] I. Bah, R. Dempsey and P. Weck, Kerr-Schild double copy and complex worldlines, JHEP 02 (2020) 180 [arXiv: 1910.04197] [INSPIRE].

[22] N. Arkani-Hamed, Y.-t. Huang and D. O'Connell, Kerr black holes as elementary particles, JHEP 01 (2020) 046 [arXiv:1906.10100] [INSPIRE].

[23] E. Lescano and J.A. Rodríguez, $\mathcal{N}=1$ supersymmetric double field theory and the generalized Kerr-Schild ansatz, JHEP 10 (2020) 148 [arXiv: 2002.07751] [INSPIRE].

[24] M.K. Gumus and G. Alkac, More on the classical double copy in three spacetime dimensions, Phys. Rev. D 102 (2020) 024074 [arXiv:2006.00552] [INSPIRE].

[25] G. Elor, K. Farnsworth, M.L. Graesser and G. Herczeg, The Newman-Penrose map and the classical double copy, JHEP 12 (2020) 121 [arXiv: 2006.08630] [INSPIRE].

[26] D.A. Easson, C. Keeler and T. Manton, Classical double copy of nonsingular black holes, Phys. Rev. D 102 (2020) 086015 [arXiv:2007.16186] [InSPIRE].

[27] H. Godazgar, M. Godazgar, R. Monteiro, D. Peinador Veiga and C.N. Pope, Weyl double copy for gravitational waves, Phys. Rev. Lett. 126 (2021) 101103 [arXiv:2010.02925] [INSPIRE].

[28] D.S. Berman, K. Kim and K. Lee, The classical double copy for M-theory from a Kerr-Schild ansatz for exceptional field theory, JHEP 04 (2021) 071 [arXiv:2010.08255] [INSPIRE].

[29] S.G. Prabhu, The classical double copy in curved spacetimes: Perturbative Yang-Mills from the bi-adjoint scalar, arXiv:2011.06588 [INSPIRE]. 
[30] C.D. White, Twistorial foundation for the classical double copy, Phys. Rev. Lett. 126 (2021) 061602 [arXiv: 2012.02479] [INSPIRE].

[31] R. Monteiro, D. O'Connell, D. Peinador Veiga and M. Sergola, Classical solutions and their double copy in split signature, JHEP 05 (2021) 268 [arXiv:2012.11190] [INSPIRE].

[32] E. Lescano and J.A. Rodríguez, Higher-derivative heterotic double field theory and classical double copy, JHEP 07 (2021) 072 [arXiv:2101.03376] [INSPIRE].

[33] M. Campiglia and S. Nagy, A double copy for asymptotic symmetries in the self-dual sector, JHEP 03 (2021) 262 [arXiv:2102.01680] [INSPIRE].

[34] G. Alkac, M.K. Gumus and M. Tek, The Kerr-Schild double copy in Lifshitz spacetime, JHEP 05 (2021) 214 [arXiv:2103.06986] [INSPIRE].

[35] E. Chacón, S. Nagy and C.D. White, The Weyl double copy from twistor space, JHEP 05 (2021) 2239 [arXiv:2103.16441] [INSPIRE].

[36] G. Alkac, M.K. Gumus and M.A. Olpak, Kerr-Schild double copy of the Coulomb solution in three dimensions, Phys. Rev. D 104 (2021) 044034 [arXiv:2105.11550] [INSPIRE].

[37] S. Angus, K. Cho and K. Lee, The classical double copy for half-maximal supergravities and T-duality, JHEP 10 (2021) 211 [arXiv:2105.12857] [inSPIRE].

[38] E. Chacón, A. Luna and C.D. White, The double copy of the multipole expansion, arXiv:2108.07702 [INSPIRE].

[39] F. Diaz-Jaramillo, O. Hohm and J. Plefka, Double field theory as the double copy of Yang-Mills, arXiv:2109.01153 [INSPIRE].

[40] R. Gonzo and C. Shi, Geodesics from classical double copy, Phys. Rev. D 104 (2021) 105012 [arXiv:2109.01072] [INSPIRE].

[41] K. Cho, K. Kim and K. Lee, The off-shell recursion for gravity and the classical double copy for currents, arXiv:2109.06392 [INSPIRE].

[42] H. Godazgar, M. Godazgar, R. Monteiro, D. Peinador Veiga and C.N. Pope, Asymptotic Weyl double copy, JHEP 11 (2021) 126 [arXiv:2109.07866] [INSPIRE].

[43] T. Adamo and U. Kol, Classical double copy at null infinity, arXiv:2109.07832 [INSPIRE].

[44] H. Stephani, D. Kramer, M.A.H. MacCallum, C. Hoenselaers and E. Herlt, Exact solutions of Einstein's field equations, Cambridge Monographs on Mathematical Physics, Cambridge University Press, Cambridge, U.K. (2003) [INSPIRE].

[45] H. Bondi, M.G.J. van der Burg and A.W.K. Metzner, Gravitational waves in general relativity. 7. Waves from axisymmetric isolated systems, Proc. Roy. Soc. London A 269 (1962) 21.

[46] Z.W. Chong, M. Cvetič, H. Lü and C.N. Pope, Charged rotating black holes in four-dimensional gauged and ungauged supergravities, Nucl. Phys. B 717 (2005) 246 [hep-th/0411045] [INSPIRE].

[47] A. Strominger, Lectures on the infrared structure of gravity and gauge theory, arXiv: 1703.05448 [INSPIRE].

[48] E.T. Newman and T.W.J. Unti, Behavior of asymptotically flat empty spaces, J. Math. Phys. 3 (1962) 891 [INSPIRE]. 
[49] A. Ilderton and D. Seipt, Backreaction on background fields: A coherent state approach, Phys. Rev. D 97 (2018) 016007 [arXiv:1709.10085] [INSPIRE].

[50] T. Adamo, L. Mason and A. Sharma, MHV scattering of gluons and gravitons in chiral strong fields, Phys. Rev. Lett. 125 (2020) 041602 [arXiv: 2003.13501] [INSPIRE].

[51] E. Witten, A note on complex spacetime metrics, arXiv:2111.06514 [INSPIRE].

[52] E. Newman and R. Penrose, An approach to gravitational radiation by a method of spin coefficients, J. Math. Phys. 3 (1962) 566 [InSPIRE].

[53] G. Barnich and C. Troessaert, Finite BMS transformations, JHEP 03 (2016) 167 [arXiv: 1601.04090] [INSPIRE].

[54] G. Compère and J. Long, Vacua of the gravitational field, JHEP 07 (2016) 137 [arXiv: 1601.04958] [INSPIRE].

[55] A. Ball, M. Pate, A.-M. Raclariu, A. Strominger and R. Venugopalan, Measuring color memory in a color glass condensate at electron-ion colliders, Annals Phys. 407 (2019) 15 [arXiv: 1805.12224] [INSPIRE].

[56] G. Barnich and R. Ruzziconi, Coadjoint representation of the BMS group on celestial Riemann surfaces, JHEP 06 (2021) 079 [arXiv: 2103.11253] [INSPIRE].

[57] M. Bianchi, F. Fucito, G.C. Rossi and M. Martellini, ALE instantons in string effective theory, Nucl. Phys. B 440 (1995) 129 [hep-th/9409037] [INSPIRE].

[58] M.J. Duff, R.R. Khuri and J.X. Lu, String solitons, Phys. Rept. 259 (1995) 213 [hep-th/9412184] [INSPIRE].

[59] A. Ball, S. Narayanan, J. Salzer and A. Strominger, Perturbatively Exact $w_{1+\infty}$ asymptotic symmetry of quantum self-dual gravity, arXiv:2111.10392 [INSPIRE].

[60] R. Britto, F. Cachazo, B. Feng and E. Witten, Direct proof of tree-level recursion relation in Yang-Mills theory, Phys. Rev. Lett. 94 (2005) 181602 [hep-th/0501052] [INSPIRE].

[61] H. Elvang and Y.-t. Huang, Scattering amplitudes, arXiv:1308.1697 [INSPIRE].

[62] J.M. Henn and J.C. Plefka, Scattering amplitudes in gauge theories, Springer, Berlin Germany (2014) [INSPIRE].

[63] R. K. Sachs, Gravitational waves in general relativity. 8. Waves in asymptotically flat space-times, Proc. Roy. Soc. London A 270 (1962) 103.

[64] G. Barnich and C. Troessaert, Aspects of the BMS/CFT correspondence, JHEP 05 (2010) 062 [arXiv: 1001.1541 ] [INSPIRE].

[65] E. Conde and P. Mao, BMS Supertranslations and Not So Soft Gravitons, JHEP 05 (2017) 060 [arXiv: 1612.08294 ] [inSPIRE].

[66] H. Lü, P. Mao and J.-B. Wu, Asymptotic structure of Einstein-Maxwell-dilaton theory and its five dimensional origin, JHEP 11 (2019) 005 [arXiv: 1909.00970] [INSPIRE].

[67] J. Ehlers and W. Kundt, Exact solutions of the gravitational field equations, in Gravitation: an introduction to current research, John Wiley \& Sons Inc., U.S.A. (1962).

[68] J.B. Griffiths and J. Podolsky, Exact space-times in Einstein's general relativity, Cambridge Monographs on Mathematical Physics, Cambridge University Press, Cambridge, U.K. (2009) [INSPIRE]. 